\title{
Robot-assisted laparoscopic resection of a huge pelvic tumor: A case report
}

\author{
Zhuomin Jia ${ }^{1^{*}}$, Xiangjun Lyu ${ }^{2^{*}}$, Yong Xu ${ }^{2}$, Rosario Leonardi ${ }^{3^{*}}, \mathrm{Xu}_{\text {Zhang }}{ }^{2}$ \\ ${ }^{1}$ Department of Urology, Military General Hospital of Beijing PLA, Beijing, 100700, China; \\ 2 Department of Urology/State Key Laboratory of Kidney Diseases, Chinese PLA General Hospital/PLA Medical School, \\ Beijing 100853, China; \\ ${ }^{3}$ Department of Urology, Musumeci GECAS Clinic, Gravina di Catania - 95030, Italy. \\ ${ }^{*}$ Contributed equally.
}

\begin{abstract}
Summary
The traditional open surgery, for the treatment of huge tumor in the narrow space of pelvic cavity and in close proximity to pelvic organs and neurovascular structures, is very difficult and challenging. We report a case of huge neurilemmoma operated using the robot-assisted laparoscopy. We used interventional pre-operation embolization to control blood supply of tumor because MRI showed the tumor had a sufficient blood supply.
\end{abstract}

KEY WORDS: Huge pelvic neurilemmoma; Pelvic robot-assisted laparoscopic surgery; Mini-invasive surgery for pelvic tumor.

Submitted 29 April 2016; Accepted 8 May 2016

\section{INTRODUCTION}

Pelvic cavity, is a narrow space closely surrounded by muscular skeletal structures. In this space urogenital organs, intestine, sigmoid colon and rectum are located. Patients with huge space-occupying lesions in this cavity may suffer from voiding or defecation dysfunctions because of rectum or bladder compression. Due to the ultra narrow space and close proximity to pelvic organs and neurovascular structures, traditional open surgery in this cavity is very difficult and challenging (1). Convalescence is usually prolonged.

Operation using Robot da Vinci because of the advantage of high-definition (HD) vision and great surgical precision can reduce the difficulty and risk of the surgical treatment. Recently, we successfully resected a huge pelvic neurilemmoma by using robot-assisted laparoscopy.

\section{Case report}

A 33-year-old male was referred to the Department of Urology of the General Hospital of People's liberation Army in February 2016 as a case of huge pelvic tumor. The tumor was found one and a half year before and wasn't treated for personal decision of the patient. During the intercurring period, the tumor stayed with no growth, although the patient was suffering from frequent difficulty in urination and defe- cation. A month ago, magnetic resonance imaging (MRI) (Figure 1) showed a huge pelvic solid neoplasm (10 x $9.7 \times 9.0 \mathrm{~cm}$ ) with sufficient blood supply. Type B ultrasound guided puncture biopsy was performed and pathology confirmed neurilemmoma.

Electrocardiogram, chest X-ray, abdominal and urological type B ultrasound were normal. During physical examination, a sharply demarcated hard and smooth neoplasm with no margin and tenderness was found above the prostate by digital rectum examination.

We decide to candidate the patient for a robot-assisted laparoscopic resection surgery. One day before the operation, interventional embolization (Figure 2) was performed to control the blood supply of the tumor.

The MRI showed that the tumor had a sufficient blood supply. During the angiography dilated right internal iliac artery was found, meaning that the tumor received its blood supply mainly from right internal iliac artery. Besides, middle sacral artery was another blood supplier for the tumor.

These blood supply arteries were embolized by gelatin sponge particles as well. Six-hour bed rest was suggested after embolization and no uncomfortable reactions were reported.

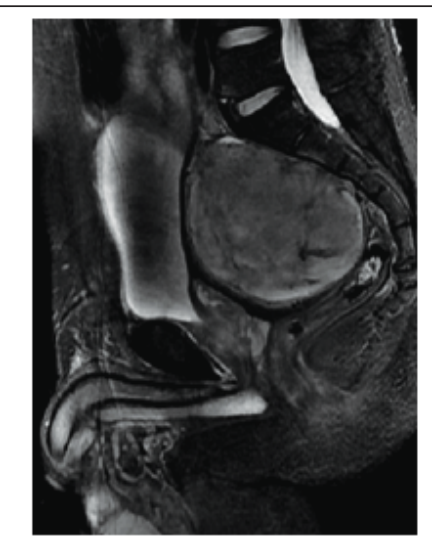

Figure 1. MRI.

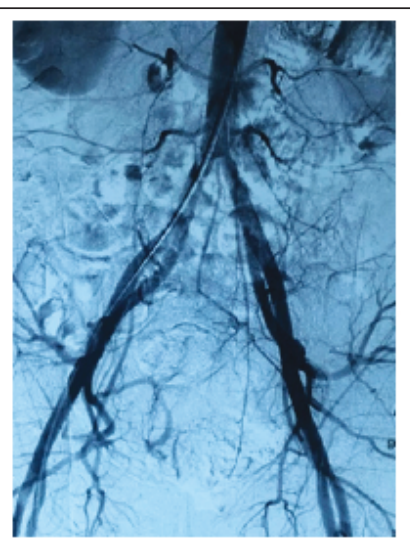

Figure 2.

Interventional embolization. 


\section{Surgical procedure}

Under general anesthesia, patient was placed in supine position, with a urinary catheter placed before the surgery. The insertion of trans umbilical scar Veress needle was used to create pneumoperitoneum. $\mathrm{CO}_{2}$ pressure was raised to $14 \mathrm{mmHg}$. A $1 \mathrm{~cm}$ incision was made about $2 \mathrm{~cm}$ up to the the umbilicus. A $12 \mathrm{~mm}$ trocar was placed in this incision for camera. Two $8 \mathrm{~mm}$ trocar were placed $8 \mathrm{~cm}$ away from the umbilicus on the left and right at umbilicus transverse line for the second and the first robotic arm, respectively. Another $8 \mathrm{~mm}$ trocar was placed $8 \mathrm{~cm}$ laterally from the right trocar for the third robotic arm. A $12 \mathrm{~mm}$ trocar was placed in the left upper abdomen as an assistant port (Figure 3). The patient was then placed in a 30 degree Trendelenburg position. Following the docking of the robotic arms, the 3-dimensional zerodegree stereoscopic endoscope, monopolar scissors (the first arm), Meryland bipolar forceps (the second arm), ProGasp forceps (the third arm) were placed for the the surgeon. Aspirator and other assistant instruments were then used by assistant through the no robotic ports.

The surgeon then moved to the console to remotely control the robot.The tumor was located in the pelvic floor, with a size of $11 \times 7 \times 7 \mathrm{~cm}$. Pelvic floor peritoneal was opened and the edge of the tumor was exposed. First was dissected the ventral surface of the tumor (Figure 4), then the left surface (Figures 5). We performed a tight dissection of left surface of tumor, along the capsula, with a high attention to not interrupt it, with an accurate hemostasis using bipolar forceps and Hem-o-lok clips as well. The right surface (Figures 6) of the tumor was closely adhesive to adjacent tissues, with sufficient blood supply. Hem-o-lok clips were used for hemostasis whereas blunt and sharp dissection was used to expose the tumor. The space between tumor and the back wall of bladder (Figure 7), tumor and the anterior rectum wall (Figure 8) was then dissected. The tumor was slight adherent to the rectum. The tumor was then successfully excised en bloc, with no rectum or bladder injury. After thorough hemostasis, silicon drainage tube was placed in the pelvic cavity, and pelvic peritoneum was closed by using Hem-o-lok clips. All robotic arms and trocars were then removed. Umbilicus incision was elongated upwards to remove the tumor. Incisions were sutured layer by layer. Total blood loss was $200 \mathrm{ml}$, total operative time, defined as the time from skin incision to skin closure was 100 minutes.

\section{Post-operative data}

The catheter was removed and patient was encouraged to get out bed one day post-operation, the gastric tube was removed 2 days post-operation, pelvic drainage tube was removed 3 days post-operation.

The patient was discharged 5 days post-operation. Postoperative pathologic examination showed neurilemmoma (Figure 10).

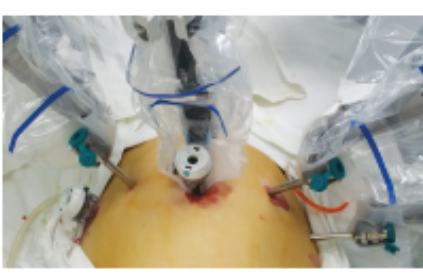

Figure 3. Trocars' position.

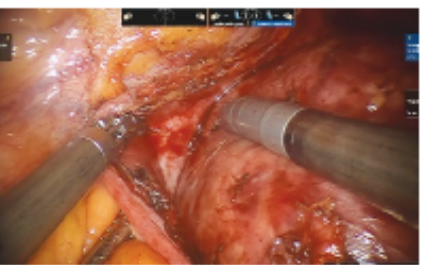

Figure 5. Left surface

of the tumor.

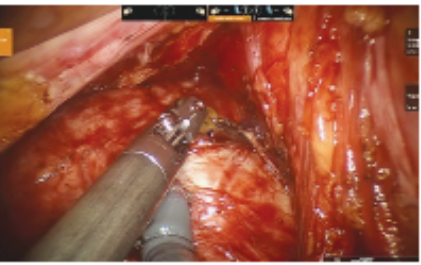

Figure 7. The space between tumor and the back wall of bladder.

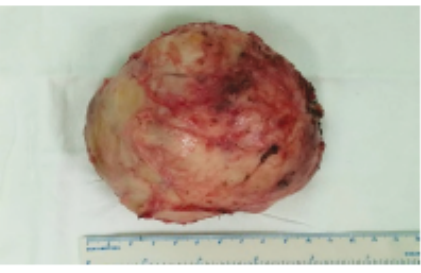

Figure 9. The tumor.

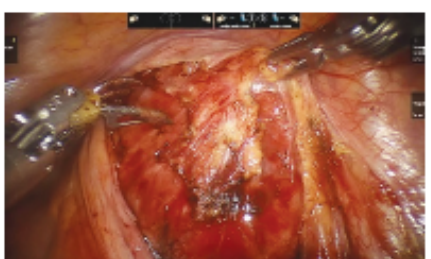

Figure 4. Ventral surface of the tumor.

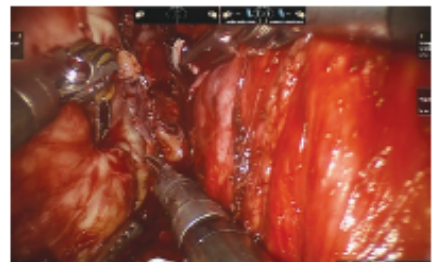

Figure 6. Right surface of the tumor.

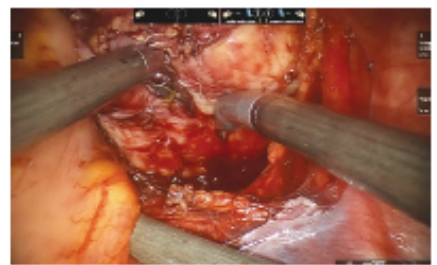

Figure 8. The space between tumor and the anterior rectum wall.

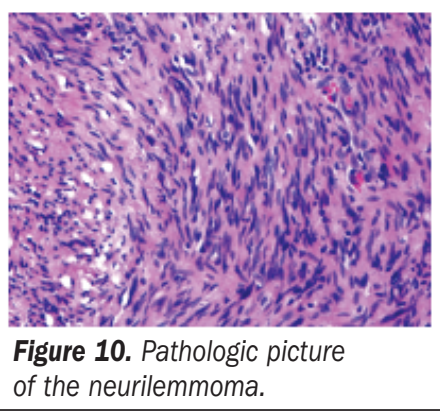

\section{Discussion}

Neurilemmoma, solitary fibrous tumor, teratoma, seminoma and extra-gastrointestinal stromal tumor are the most common pelvic tumors among males (2). The histological type of this case was neurilemmoma, which is a kind of benign tumor belong to nerve sheath tumor; another two kinds of nerve sheath tumor are neurofibroma and malignant peripheral nerve sheath tumor. These tumors are usually seen in head, neck, mediastinum, and upper limbs, but rarely, about 0.5\%-3\%, in retroperitoneal space. Pelvic neurilemmoma is even more rare, usually located in presacral space and peri-lumbosacral vertebral region, sometimes arising from pelvic wall, bladder and bowel wall (3).

Deep location, narrow space, multiple adjacent organs and bony structure of pelvic wall add difficulties to open surgery for treatment of pelvic tumors. Besides, open surgery has the disadvantage of severe operation trauma, which leads to slow recovery. In this case, the robotic surgical system DaVinciSI was used, because this system could provide surgeons with enhanced visual control and dexterity. The 3-dimensional monitor system provides us 
high-definition, magnified images to better observe tissue boundaries and vessels, which adds the possibility of sharp dissection and the possibility to remove all the tumor without interruption of the capsule and without leaving tumoral tissue that could give a relapse of pathology. The robotic arms of the system are more flexible than human hands, especially for deep-space surgeries, and the third arm works as an assistant and can be manipulated by the surgeon, making the cooperation closer. Less trauma and fast recovery are the other advantages of this system. The huge pelvic tumor of this patient was closely adjacent to bladder and rectum, and had sufficient blood supply. Thus we first controlled its blood supply prior operation by using interventional embolization to reduce the risk of intra-operative bleeding. During the operation, the relationship between the tumor and adjacent tissues could be divided into five steps, that are abdominal, left pelvic wall, right pelvic wall, back bladder wall and front rectum wall.
Robotic assisted laparoscopic surgery has the advantages of enhanced dexterity, high-definition 3-dimentional magnified images, less trauma and fast post-operative recovery in resection of huge pelvic tumor, and could be considered as an alternative procedure for treatment of huge pelvic tumor.

\section{REFERENCES}

1. Wong KC, Sze KY, Wong IO, et al. Patient-specific instrument can achieve same accuracy with less resection time than navigation assistance in periacetabular pelvic tumor surgery: a cadaveric study. Int J Comput Assist Radiol Surg. 2016; 11:307-16.

2. Daneshmand S, Youssefzadeh D, Chamie K, et al. Benign retroperitoneal schwannoma: a case series and review of the literature. Urology. 2003; 62:993-7.

3. Hoarau N, Slim K, Da ID. CT and MR imaging of retroperitoneal schwannoma. Diagn Interv Imaging. 2013; 94:1133-9.

\section{Correspondence}

Zhuomin Jia, MD

Department of Urology, Military General Hospital of Beijing PLA,

Beijing 100700, China

Xiangjun Lyu, MD

Yong Xu, MD

Xu Zhang, MD (Corresponding Author)

xzhang@foxmail.com

Department of Urology/State Key Laboratory of Kidney Diseases, Chinese PLA General Hospital/PLA Medical School,

Beijing 100853, China

Rosario Leonardi, $M D$

leonardi.r@me.com

Department of Urology, Musumeci GECAS Clinic,

Gravina di Catania - 95030, Italy 